\title{
The MASCOT Magnetometer
}

\author{
David Herčík ${ }^{1}$ (D) Hans-Ulrich Auster ${ }^{2}$ Jürgen Blum² • Karl-Heinz Fornaçon² • \\ Masaki Fujimoto ${ }^{3}$ - Kathrin Gebauer ${ }^{2}$ - Carsten Güttler ${ }^{4}$. Olaf Hillenmaier ${ }^{5}$. \\ Andreas Hördt ${ }^{2}$ - Evelyn Liebert ${ }^{2}$ - Ayako Matsuoka ${ }^{3} \cdot$ Reiko Nomura $^{3}$. \\ Ingo Richter ${ }^{2} \cdot$ Bernd Stoll $^{2} \cdot$ Benjamin P. Weiss $^{6} \cdot$ Karl-Heinz Glassmeier $^{2}$
}

Received: 21 August 2015 / Accepted: 8 January 2016 / Published online: 26 January 2016

(C) The Author(s) 2016. This article is published with open access at Springerlink.com

\begin{abstract}
The Mobile Asteroid Scout (MASCOT) is a small lander on board the Hayabusa2 mission of the Japan Aerospace Exploration Agency to the asteroid 162173 Ryugu. Among the instruments on MASCOT is a fluxgate magnetometer, the MASCOT Magnetometer (MasMag). The magnetometer is a lightweight $(\sim 280 \mathrm{~g})$ and low power $(\sim 0.5 \mathrm{~W})$ triaxial fluxgate magnetometer. Magnetic field measurements during the landing period and during the surface operational phase shall provide information about any intrinsic magnetic field of the asteroid and its remanent magnetization. This could provide important constraints on planet formation and the thermal and aqueous evolution of primitive asteroids.
\end{abstract}

Keywords Magnetometer $\cdot$ MASCOT $\cdot$ Hayabusa2 $\cdot$ Asteroids

\section{Introduction}

The Mobile Asteroid Surface Scout (MASCOT) (Ho et al., this issue) is a small lander on the Hayabusa2 mission of the Japan Aerospace Exploration Agency (JAXA) bound for the near-Earth asteroid 162173 Ryugu (provisional designation $\left.1999 \mathrm{JU}_{3}\right)($ Campins et al. 2013). The Hayabusa2 mission will provide in situ and remote observations of the asteroid

D. Herčík

d.hercik@tu-bs.de

1 Institut für Geophysik und extraterrestrische Physik, Technische Universität Braunschweig, Mendelssohnstr. 3, 38106 Braunschweig, Germany

2 Institut für Geophysik und extraterrestrische Physik, Technische Universität Braunschweig, Braunschweig, Germany

3 Institute of Space and Astronautical Science, Japan Aerospace Exploration Agency, Kanagawa, Japan

4 Max-Planck-Institut für Sonnensystemforschung, Göttingen, Germany

5 Magson GmbH, Berlin, Germany

6 Department of Earth, Atmospheric and Planetary Sciences, Massachusetts Institute of Technology, Cambridge, MA, USA 
and will then return samples to Earth. The MASCOT lander will provide in situ observations of the surface morphology, surface temperature, and magnetic field. The MASCOT payload comprises a camera (CAM) (Jaumann et al., this issue), a radiometer (MARA) (Grott et al., this issue), an infrared spectrometer (MicrOmega) (Bibring et al., this issue), and a magnetometer (MasMag). The measurements will also provide context for orbital instrumentation and derived science.

The Hayabusa2 spacecraft was launched on December 3, 2014, from Tanegashima Space Center in Japan. A cruise phase will last until mid-2018, when the spacecraft will arrive at the target asteroid 162173 Ryugu. The first observational period will be dedicated to the global mapping of the asteroid to gain more detailed information about the shape, gravity, and surface properties. A date for the MASCOT lander release is not yet fixed and will depend on the results of the global mapping campaign, particularly on the shape and the thermal conditions of the asteroid.

The target is a Cg-type asteroid belonging to the Apollo group of the near-Earth asteroids. Many carbonaceous asteroids are thought to be primitive bodies that have escaped intense thermal metamorphism and have undifferentiated exteriors. They may therefore contain relatively pristine materials that formed in the solar nebula and record the formation and the evolution of the early solar system. Their material properties are derived from investigations of carbonaceous chondrites and the identification of C-types asteroids as their presumed parental bodies (Burbine et al. 2002). Carbonaceous chondrites from the $\mathrm{CV}$ and $\mathrm{CM}$ groups are known to contain natural remanent magnetization, indicating that a magnetic field was present early in the history of the parental body, either during the formation or later as a product of an internal dynamo (Weiss et al. 2010; Cournede et al. 2015). The natural remanent magnetization of chondrites has been found to very widely vary from $10^{-4}$ to $10^{-2} \mathrm{Am}^{2} / \mathrm{kg}$ (Terho et al. 1996; Gattacceca and Rochette 2004), with most measurements above $10^{-3} \mathrm{Am}^{2} / \mathrm{kg}$, likely reflecting remagnetization by collectors' hand magnets on Earth.

Magnetic fields may have played an important role in the solar system formation. For example, it has been proposed that dust coagulation was enhanced for magnetized particles by inter-particular magnetic forces (Nübold and Glassmeier 2000). On the other hand, a large-scale magnetic field of the order of $10^{-5}$ to $10^{-4} \mathrm{~T}$ in the solar nebula could be able to align particles to form up to centimeter-sized or larger conglomerates with coherent magnetization (Fu and Weiss 2012).

The detection of such large-scale magnetization could therefore provide evidence for a nebular field, which has been proposed to play an essential role in mass and momentum transfer in the protoplanetary disk (Turner et al. 2014). In fact, recent paleomagnetic studies of the LL ordinary chondrite Semarkona chondrite indicate that a nebular field of 5-50 uT field was present at 2-3 million years after the formation of the solar system (Fu et al. 2014). Measurements of the spatial scale of magnetization in an asteroid could distinguish between various processes proposed for concentrating rocky materials into selfgravitating clumps that could collapse to form planetesimals. Finally, it has been proposed that some early planetesimals may have partially differentiated, forming a molten metallic core that magnetized an overlying unmelted chondritic crust (Elkins-Tanton et al. 2011; Carporzen et al. 2011); this hypothesis could be tested by searching for remanent magnetization produced after the dissipation of the solar nebula.

Direct in situ observations of the primitive bodies are scarce. Global magnetization values have been estimated from flybys of several asteroids (Table 1). The estimated values of net dipole moments and specific magnetic moments are given here in orders of magnitude only to provide an overview range. In the case of Gaspra, a dipole moment has been derived 
Table 1 Data used from Kivelson et al. (1993), Richter et al. (2001, 2012), Auster et al. (2010), Acuña et al. (2002), Coradini et al. (2011), McCoy et al. (2001). The magnetization values for simplicity show orders of magnitude only

\begin{tabular}{llllll}
\hline Asteroid & Gaspra & Braille & Šteins & Lutetia & Eros \\
\hline Mean radius $[\mathrm{km}]$ & 7 & 0.8 & 3 & 49 & 9 \\
Density $\left[\mathrm{kg} / \mathrm{m}^{3}\right]$ & 4000 & 3900 & 3200 & 3400 & 2650 \\
Material & Metal & Basalt & Enstatite & Chondrites & Chondrites \\
& Olivine & Olivine & & Enstatite & Achondrites \\
& Pyroxene & Pyroxene & & & 12.2 .2001 \\
Fly-by & 29.10 .1991 & 29.7 .1999 & 5.9 .2008 & 10.7 .2010 & landing \\
CA $[\mathrm{km}]$ & 1600 & 28 & 799 & 3120 & $<5$ \\
Measured field $[\mathrm{nT}]$ & draping & 2 & $<1$ & $<1$ & $<10^{10}$ \\
Estimated values & & & & $<10^{12}$ & $<10^{-6}$ \\
Dipole moment $\left[\mathrm{Am}{ }^{2}\right]$ & $\sim 10^{13}$ & $\sim 10^{11}$ & $<10^{12}$ & $<10^{-3}$ & $<1$ \\
Spec. moment $\left[\mathrm{Am}{ }^{2} / \mathrm{kg}\right]$ & $\sim 10^{-2}$ & $\sim 10^{-2}$ & $<10^{-3}$ & & $<$ \\
\hline
\end{tabular}

from measurements of a draping effect of a magnetic field observed by the Galileo spacecraft during a fly-by (Kivelson et al. 1993). However, as Blanco-Cano (2003) argued, the effect could have been created as well by the solar wind itself. Richter et al. (2001) reported on measurements with the Deep Space 1 magnetometer during the Braille fly-by. The observations show a $\sim 2 \mathrm{nT}$ increase in magnetic field at the closest approach. Measurements from Šteins (Auster et al. 2010) and Lutetia (Richter et al. 2012), on the other hand, revealed no observable increase in the magnetic field, and the estimated values of magnetization are upper limits only. These examples suggest that the determination of magnetization from a distant fly-by is extremely difficult.

A more reliable source of information is provided by direct in situ measurements from the surface. There are, so far, only two such measurements: the landing of the Near Earth Asteroid (NEAR)-Shoemaker spacecraft (Veverka et al. 2001; Acuña et al. 2002) on Eros, and the landing of Philae (Biele and Ulamec 2008; Auster et al. 2015) on the nucleus of comet 67P/Churyumov-Gerasimenko.

For Eros, an S-type asteroid, no detectable intrinsic magnetic field was observed from orbit or on the surface. An upper limit on the global specific magnetic moment of $<2 \times$ $10^{-6} \mathrm{Am}^{2} / \mathrm{kg}$ was derived from the $\sim 5 \mathrm{nT}$ measurement accuracy (Acuña et al. 2002).

No intrinsic magnetic field was detected with the ROMAP instrument (Auster et al. 2015) during the landing of Philae on 67P/Churyumov-Gerasimenko. Because of the unlikely case of the global magnetization, the authors did provide magnetization depending on a scale of magnetic heterogeneity instead. For randomly oriented dipoles centered in $1 \mathrm{~m}^{3}$ blocks with internally uniform magnetization, they estimate an upper limit on the specific magnetic moment of $<3 \times 10^{-5} \mathrm{Am}^{2} / \mathrm{kg}$. The estimate is based on a measurement uncertainty of the DC surface field of $<2 \mathrm{nT}$ and measurement height of $0.4 \mathrm{~m}$.

The MASCOT magnetometer (MasMag) will provide the first magnetic field measurements at the surface of a C-type asteroid and the magnetic transect across the surface of an asteroid. Combined with anticipated laboratory magnetic studies of the asteroid samples after return to Earth, it will be the most comprehensive magnetic investigation of a small body to date. Here we provide details on the scientific objectives, the instrument design, on-ground calibrations, and first commissioning results. 

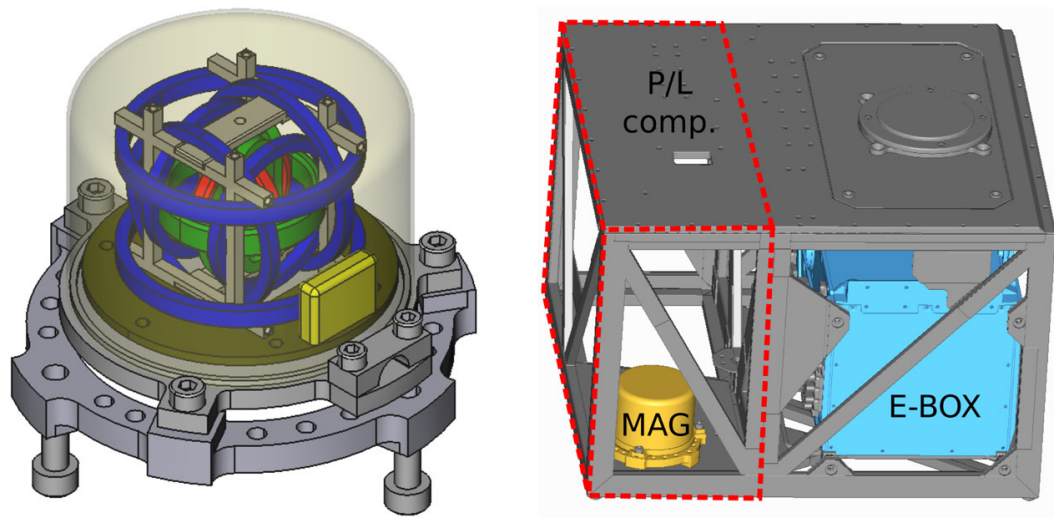

Fig. 1 Left: MasMag sensor inner structure with cores and excitation coils (red), pick-up coils ( green), and feedback Helmholz coil system (blue). Right: position of the MasMag sensor within the lander body frame. All the payload instrument sensors are accommodated in the payload (P/L) compartment (indicated by the red dashed line); for better visibility, only the MasMag sensor is shown here. The electronics boards are placed within the electronic box (E-BOX) (light blue). The MASCOT battery (not shown here) is accommodated on top of the E-BOX. The whole MASCOT structure has dimension of $\sim 20 \times 30 \times 30 \mathrm{~cm}^{3}$. The MasMag sensor without the stand-off is $\sim 55 \mathrm{~mm}$ in diameter and $\sim 50 \mathrm{~mm}$ in height

\section{Science Objectives}

In summary, measurements of remanent magnetization of primitive bodies like asteroids and comets may provide records of the solar nebula magnetic field and of small-body dynamos. MasMag measurements of asteroid Ryugu offer the possibility of inferring the intensity of the nebular field at a distinct location and time in the early solar system not represented by the other bodies analyzed in detail so far (i.e., the S-type asteroid 433 Eros, comet 67P, and the LL, CV, and CM chondrite parent bodies).

MASCOT provides a suitable platform for such observations. MasMag is placed inside the MASCOT lander body on the bottom panel (Fig. 1). This mounting provides a suitable measurement situation as the sensor is very close to the surface. However, this location is also challenging in terms of magnetic cleanliness issues and contamination of the asteroidal magnetic field by spacecraft-generated bias fields.

Single-point measurements are difficult to generalize. MASCOT has the ability to relocate itself on the surface of the asteroid thanks to a built-in mobility mechanism consisting of a movable arm with an eccentric mass (Reill et al. 2015). The momentum transfer from the arm to the lander body will enable MASCOT to upright itself to a default operational position and also to hop to another place on the asteroid (up to $\sim 100 \mathrm{~m}$ distant). The relocation will provide a unique opportunity to measure magnetic fields at several locations, enabling higher confidence levels for the measurements as well as magnetic field gradient information. Investigation of the magnetic field during the descent and hopping will provide information on the spatial decay of the field from which useful information about a global field or local sources might be obtained.

The scientific objective is therefore to measure any global as well as local magnetic field during the whole MASCOT operational period, focusing on landing and relocation phases.

The quantitative requirements can be defined based only on the limited knowledge from previous magnetic field observations of primitive bodies, such as shown in Table 1 or from the above-mentioned Philae landing on the comet. The MasMag shall ensure a measurement 
Table 2 MasMag parameters and performance

\begin{tabular}{ll}
\hline Parameter & Value \\
\hline Sensor mass [g] & 89 \\
Electronics mass [g] & 180 \\
Power consumption [W] & 0.5 \\
Sensor dimensions $\left[\mathrm{mm}^{3}\right]$ & $\pi \times 27.5^{2} \times 50$ \\
Electronics dimensions $\left[\mathrm{mm}^{3}\right]$ & $109 \times 94 \times 20$ \\
Dynamic range [nT] & \pm 12000 \\
Sensor noise @ $1 \mathrm{~Hz}[\mathrm{pT} / \sqrt{\mathrm{Hz}}]$ & $<15$ \\
Resolution [pT] & 2 \\
Sampling rate $[\mathrm{Hz}]$ & 10 \\
Nominal data volume $[\mathrm{bit} / \mathrm{s}]$ & 1010 \\
\hline
\end{tabular}

accuracy of $1 \mathrm{nT}$ in order to be able to also estimate local magnetization of the surface material within a $10^{-5} \mathrm{Am}^{2} / \mathrm{kg}$ specific moment range for decimeter-sized magnetic domains granularity. The sensitivity requirement shall be better than $0.1 \mathrm{nT}$ in order to be able to identify signal variation for the magnetic cleanliness analysis. As the scientific objectives do not include specific plasma physics wave phenomena, the required data rate is driven by the need of resolution of sharp field changes, indicating artificial signal. The jumps in data shall be well resolved within a $1 \mathrm{~s}$ period. Therefore, a $10 \mathrm{~Hz}$ data sampling rate is sufficient for the magnetometer.

\section{Instrument Description}

The MASCOT magnetometer instrument is a vector-compensated three-axis fluxgate magnetometer consisting of a sensor head and digital electronics. The instrument has a long heritage from various space missions such as THEMIS (Auster et al. 2008), Venus Express (Zhang et al. 2007), ROSETTA (Auster et al. 2007) or BepiColombo (Glassmeier et al. 2010). The sensor itself has been built at the Institut für Geophysik und extraterrestrische Physik (IGeP), Technische Universität Braunschweig in cooperation with Magson GmbH (Berlin) providing the electronics.

The MasMag electronics board (PCB) is placed inside MASCOT's common electronics box (E-BOX) (Ho et al., this issue), while the sensor itself is mounted near a corner of the lander's body frame (Fig. 1). Above the magnetometer sensor, the camera instrument (CAM) is mounted. The payload compartment is furthermore occupied by the radiometer (MARA) and the spectrometer (MicrOmega). The four instruments are designed for a detailed characterization of the asteroid surface.

The magnetometer will measure three components of a magnetic field with a $10 \mathrm{~Hz}$ sampling rate. The performance parameters are summarized in Table 2.

\subsection{Sensor}

The magnetometer sensor (Fig. 2) resides in the payload compartment of the lander as depicted in Fig. 1 (right panel). The sensor is mounted on the bottom plate of the MASCOT structure. The sensor head is attached via bolts to a ring-shaped stand-off made of PEEK and connected to the electronics card located in the MASCOT E-BOX via a pigtail harness. 


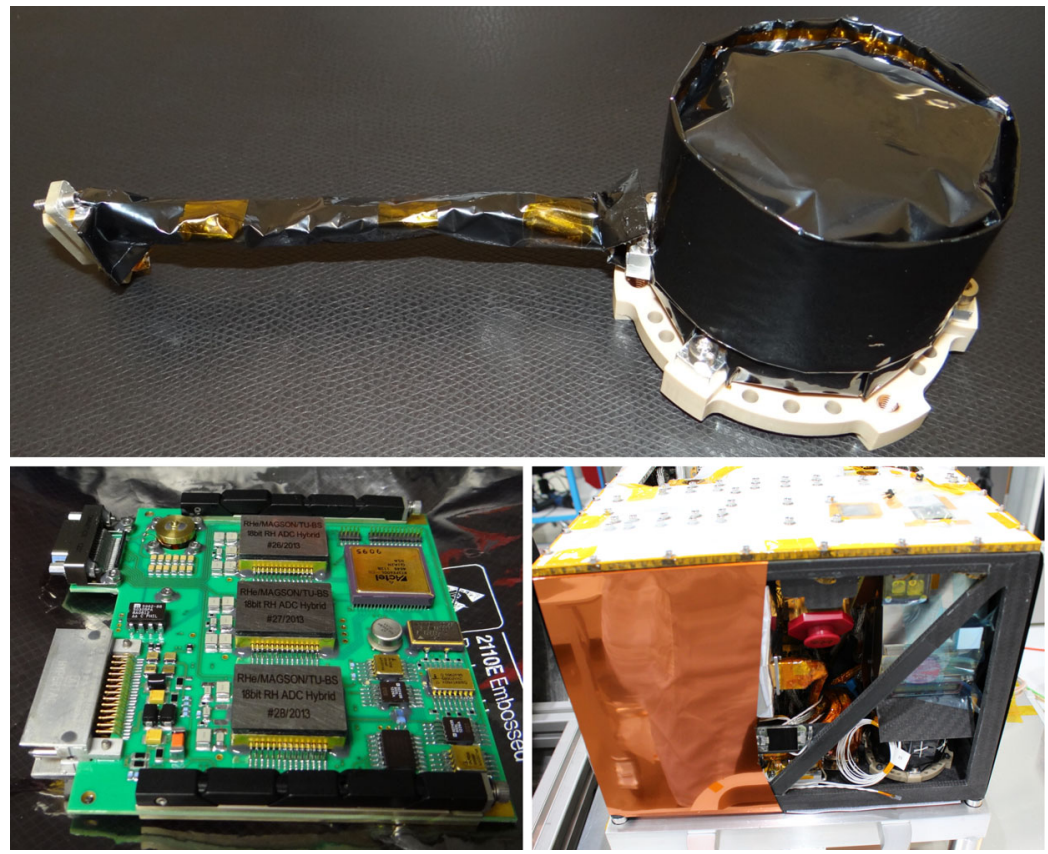

Fig. 2 Top: The MasMag sensor with a $10 \mathrm{~cm}$-long pigtail harness, all wrapped in black MLI. Bottom left: The MasMag PCB $\left(\sim 10 \times 10 \mathrm{~cm}^{2}\right)$. Bottom right: The MASCOT lander during the integration with the MasMag sensor mounted in the right bottom corner below the camera instrument

The principle of operation of a fluxgate-type magnetometer (Auster 2008) is based on periodic positive and negative saturation of a highly permeable material. An excitation current driven with a specific excitation frequency through a coil wound around the core material produces an alternating signal in the core that is picked up by a secondary coil. According to the fluxgate principle, an external magnetic field distorts the symmetry of the magnetic flux inside the core and generates a signal in even harmonics of the excitation frequency proportional to the external field. A sense coil picks up the external field component in the direction of the coil axis. By proper design of a system of three perpendicular secondary coils, complete information about the direction and magnitude of the external field can be extracted.

The MasMag sensor design (Fig. 1, left panel) consists of two ring core elements of highpermeability material (mu-metal) that serves as the external magnetic field concentrator. The excitation coils are wound tightly around the ring cores. A second set of coils- the three axes sensing coil system-picks up the induced signal. Additionally, a complete Helmholtz coil system is mounted within the sensor to provide feedback and to maintain the center of the sensor in a near-zero field. This feature keeps the sensor in a linear regime and avoids the need of range switching. Information about the ambient magnetic field is then extracted using both the sensed signal and the feedback current values. The sensor coil system is encapsulated in a cylindrical aluminum shell and connected to the electronics via a $\sim 10 \mathrm{~cm}$ long pigtail harness.

The payload compartment of the lander is thermally uncontrolled. Therefore, the sensor is covered by multi-layered insulation (MLI) (Fig. 2) to achieve maximum thermal stability. The thermal control of the sensor is completely passive through good insulation from exte- 
rior influences. The temperature of the sensor is monitored by the magnetometer electronics. An 8-layered MLI with black Kapton on the outer side is used for thermal insulation. The black outer layer has been selected upon request from the camera instrument team to avoid reflections from the MLI surface to the optics of the camera.

\subsection{Electronics}

The instrument electronics board (Fig. 2) is placed in the MASCOT common E-BOX together with other system and payload electronic cards. The front-end electronics of MasMag provides overall control of the magnetometer and communication with the MASCOT on-board computer (OBC).

The instrument is fully controlled by a single Microsemi RT3PE600L FPGA. It provides functions for computation of the measured field and control over the feedback current. It handles the communication and switches the instrument into different operational modes. The sensor electronics generates an excitation AC current (fundamental excitation frequency of approx. $9.6 \mathrm{kHz}$ ). The induced voltage in the sense coil is digitized immediately behind the preamplifier at a cadence four times higher than the excitation frequency. Although the 2nd harmonics frequency that holds the information about the ambient magnetic field is equal to the Nyquist frequency, the problem of aliasing is mitigated by the implementation of a band-pass filter around the 2nd harmonics prior to the digitization process, which ensures the instrument's withstanding higher frequencies. The front-end signal processing (synchronous detection and integration as well as the feedback value and the field calculation) is accomplished by logic blocks within the field-programmable gate array (FPGA). The feedback field improves the overall linearity and stability of the magnetometer. It is supplied to all sensor elements via a set of two 16-bit digital-to-analogue converters (DACs) (feedback DACs) and a separate pair of feedback coils (Helmholtz coils) per sensor axis. The sense from the analogue-to-digital converter (ADC) and the feedback values (setting of feedback DACs) are continuously used for calculating the magnetic field values (24 bit) by scaling and adding: $k 1 \cdot A D C+k 2 \cdot D A C F+k 3 \cdot D A C C$. Here $k 1, k 2$, and $k 3$ are coefficients set during the instrument development and the testing procedure and used as weights for the field computation. The $A D C$ stands for a value resulting from the analogue-to-digital converter from the sense coil signal; $D A C$ is the feedback value on the digital-to-analogue converter. The feedback of the current design is composed of two DACs: coarse (DACC) for the field compensation by larger steps and fine $(D A C F)$ for finer compensation with a better resolution. This allows an artificial field to be applied to the sensor via the feedback loop to compensate both ambient and bias field. The approach of combining one 18-bit ADC and two 16-bit DAC values into the magnetic field computation enables single measurement range while having a sufficient resolution (24-bit). Moreover, the DACC can be used not only for the dynamic feedback, but can also be set to a static value to compensate static bias field.

Although the digital magnetometer concept requires an analogue-to-digital conversion at a high-data rate, it exhibits a number of advantages over the more traditional analogue fluxgate magnetometer. Early digitization makes the sensed signal robust with respect to changes of the environmental temperature and supply voltages, as well as insensitive to electro-magnetic disturbances. Furthermore, no range switching is needed to achieve the required resolution, which reduces the complexity of design and data analysis.

For these types of magnetometers, a special analogue-to-digital converter hybrid circuit has been developed by Magson GmbH and IGeP. The hybrid circuit implements a preamplifier and an ADC with a latch-up protection into a single electronic chip that enables easy 
spot shielding. The ADC hybrids have been integrated and manufactured by the RHe Microsystems $\mathrm{GmbH}$ (Radeberg). To enhance the radiation tolerance, titanium and wolfram shielding is attached to the top and bottom of the chip package. MasMag therefore will provide proof-of-flight for this new technology that will be used on future space missions such as the Jupiter Icy Moons Explorer (JUICE) (Grasset et al. 2013).

A processor system, which is an in-house development by Magson $\mathrm{GmbH}$, is used for processing the magnetometer data and managing the interface with OBC. It contains a reduced instruction set computing (RISC)-like processor according to Harvard architecture, a computer architecture with physically separate storage for data and instructions allowing for parallel access to the code and data memory. The processor is highly configurable in its parameters, such as data and instruction width, and can easily be adapted to the processing requirements of a specific project. In most applications, it is used with a data width of 32 bits and an instruction width of 16 bits. It is programmed using a machine-oriented assembly language with 19 instructions. The processor was originally developed for the NASA Themis mission (Angelopoulos 2008), and since then it has continuously been improved and expanded in its functionality. The MasMag instrument is supplied by a MASCOT power conversion and distribution unit (PCDU) with the regulated voltages of $3.3 \mathrm{~V}$, $+5 \mathrm{~V}$, and $-5 \mathrm{~V}$. The MasMag instrument uses an EIA422-compatible serial interface. The interface consists of a quad receiver and quad transmitter on the MasMag PCB, providing two equivalent interfaces, while two receivers and transmitters remain unused. MasMag listens to both equivalent interfaces and replies to the one from which a valid telecommand (TC) was received. OBC is connected to both interfaces, but only one is enabled at a time. The data interface is a low-speed universal asynchronous receiver/transmitter (UART) with a data transmission rate of $19.2 \mathrm{kbps}$.

\subsection{Ground Tests and Calibration}

The magnetometer was subjected to a series of qualification and calibration procedures as a stand-alone instrument as well as an integrated part of the whole MASCOT. An extensive effort was spent on a magnetic characterization of the MASCOT lander itself. Magnetic cleanliness tests were performed on MASCOT to characterize the AC and DC signature of the lander.

The qualification on an instrument level involved vibration and thermal tests to ensure compatibility with the mission requirements. Furthermore, the instrument went through a series of thermal vacuum tests on the system level (integrated within MASCOT). For full performance of the instrument, MasMag was calibrated in the Magnetsrode facility of IGeP (Glassmeier et al. 2007). This facility is a specially built laboratory for magnetometer calibrations located outside the city of Braunschweig and far away from major magnetic disturbers such as railways. A real-time monitoring of the Earth's field is provided by an external fluxgate magnetometer and an Overhauser magnetometer. A large Helmholtz coil system ensures controlled field conditions at the tested sensor position in the center of the coil system. Additionally, a temperature chamber enables testing and calibration in a wide range of operating temperatures. Figure 3 shows the facility with the coil system. The MasMag sensor was placed within a thermal chamber (Fig. 4), allowing the sensor to be tested at various temperatures in a range from -80 to $+80^{\circ} \mathrm{C}$.

The calibration procedure includes the determination of the instrument performance in terms of the internal DC offset, the sensitivity, the internal misalignment, and the corner frequency. Based on the calibration results, a model of the dependence of instrument pa- 
Fig. 3 The Magnetsrode calibration facility, a 2-m large Helmholtz coil system. A non-magnetic temperature chamber is placed in the center of the system

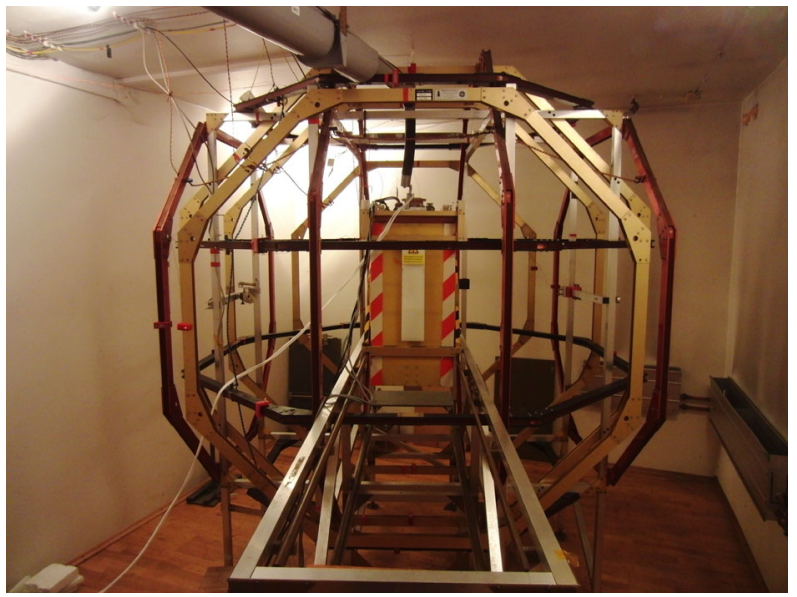

Fig. 4 The MasMag sensor placed within the temperature chamber on a rotational platform for calibrations in the Magnetsrode facility

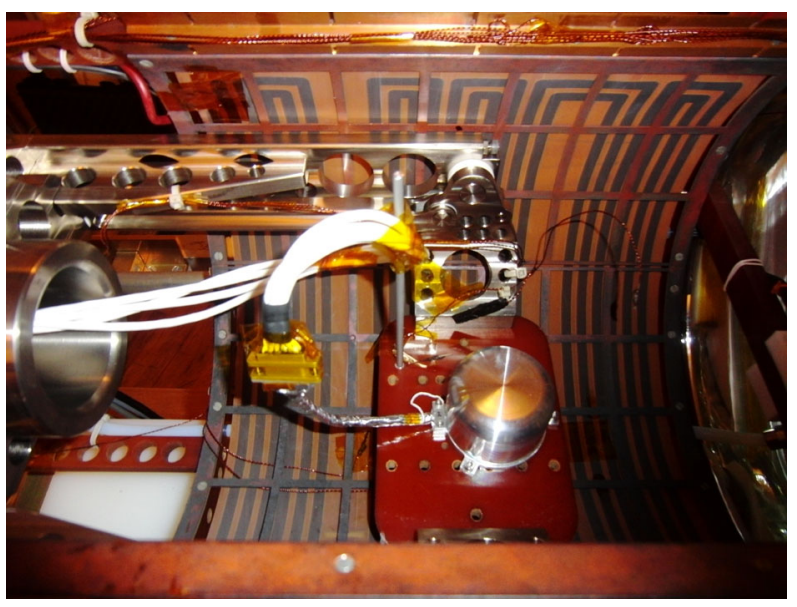

rameters on temperature is created. The model is a linear approximation of the thermal dependence in the following form:

$$
P_{i}=F_{i}^{(0)}+F_{i}^{(1)} T,
$$

where $P$ is a specific calibration parameter, $i$ is the instrument magnetic axis for which the parameter is valid, $F_{i}^{(0)}$ and $F_{i}^{(1)}$ are zero- and first-order factors as derived from the calibration, and $T$ is the sensor temperature in ${ }^{\circ} \mathrm{C}$. The model parameters and factors are given in Table 3 . The calibration parameters are implemented in a calibration matrix $\Phi$. The calibrated field $\mathbf{B}^{c}$ is then computed by applying the calibration matrix to the data measured by the magnetometer sensor $\mathbf{B}^{\mathrm{r}}$ after removal of the sensor offset $\mathbf{B}^{\text {off }}$.

$$
\mathbf{B}^{\mathrm{c}}=\boldsymbol{\Phi}\left(\mathbf{B}^{\mathrm{r}}-\mathbf{B}^{\text {off }}\right)=\rho \omega \sigma\left(\mathbf{B}^{\mathrm{r}}-\mathbf{B}^{\text {off }}\right),
$$

where $\rho$ is the rotation from the calibration facility coordinate system to ideal magnetometer sensor coordinates, $\omega(T)$ describes the temperature-dependent internal sensor misalignment, and $\sigma(T)$ represents the temperature-dependent sensitivity. 
Table 3 Factors for temperature dependence computation of the calibration parameters

\begin{tabular}{llrl}
\hline Parameter & Factor & \multicolumn{1}{c}{$F^{(0)}$} & \multicolumn{1}{l}{$F^{(1)}$} \\
\hline -3 dB corner frequency [Hz] & $f_{3 d B, x}$ & 5.478 & $-6.741 \times 10^{-3}$ \\
& $f_{3 d B, y}$ & 5.367 & $-4.054 \times 10^{-3}$ \\
& $f_{3 d B, z}$ & 5.447 & $-5.382 \times 10^{-3}$ \\
Misalignment [deg] & $\xi_{x y}$ & 89.684 & $-1.037 \times 10^{-5}$ \\
& $\xi_{x z}$ & 89.717 & $6.957 \times 10^{-5}$ \\
& $\xi_{y z}$ & 90.113 & $2.198 \times 10^{-5}$ \\
Offset [nT] & $B_{\text {off }, x}$ & 17.645 & $-1.254 \times 10^{-2}$ \\
& $B_{\text {off, } y}$ & 14.651 & $5.572 \times 10^{-3}$ \\
& $B_{\text {off, }, z}$ & 4.061 & $-6.072 \times 10^{-3}$ \\
Sensitivity [] & $\sigma_{x}$ & 0.988 & $-1.787 \times 10^{-5}$ \\
& $\sigma_{y}$ & 0.994 & $-1.342 \times 10^{-5}$ \\
& $\sigma_{z}$ & 0.991 & $-1.812 \times 10^{-5}$ \\
\hline & & & \\
& & &
\end{tabular}

\subsection{Magnetic cleanliness}

For a magnetometer, the magnetic cleanliness of the spacecraft is of highest importance. On major missions such as Cluster (Escoubet et al. 1997), a magnetic cleanliness program monitors the usage of magnetic materials and design of cable paths during the development phase. In addition, magnetometers are usually mounted on a boom to be located farther away from disturbing sources, preferably accommodating two sensors for a dual technique (Ness et al. 1971) that enables separation of the ambient and the artificial field. However, in the case of MASCOT, no such option was available due to size limitations and mass constraints. Therefore, MasMag is accommodated directly within the lander structure in the vicinity of all other payloads and system sub-units.

During the development phase, attention was paid to check the DC magnetic properties of each sub-unit. A magnetic cleanliness facility, available at IGeP, was used for that purpose. Already early in the project, a potential strong source of magnetic field was identified in the mobility mechanism. The eccentric arm contained permanent magnets that allow for a position check by Hall sensors. The original design would lead to several thousands of nT at the MasMag sensor position, an unacceptable value. A solution was found by reversing one of the two magnets into an anti-parallel orientation. This self-compensating setup was experimentally demonstrated to produce a magnetic field of only 50-100 nT at the MasMag sensor position.

Another investigation revealed a high magnetization for the MASCOT battery provided by the Centre National d'Etudes Spatiales (CNES). Measurements indicate a dipole moment of $>430 \mathrm{mAm}^{2}$. CNES therefore established a demagnetization procedure that decreased the magnetization to around $\sim 5 \mathrm{mAm}^{2}$. In spite of the major efforts that successfully reduced the existing disturbances, the DC magnetic field from the whole MASCOT still cannot be neglected. This holds for the AC signals produced by operations of units as well. Therefore, dedicated tests were carried out in order to characterize the magnetic signature of the fully integrated MASCOT.

The first test of the series took place with the flight model (FM) at the German Aerospace Center (DLR) in Bremen, using a single mu-metal shield tube as a magnetic field shielding. The second and third tests were performed on the Sagamihara campus of the Japan 
Aerospace Exploration Agency (JAXA) using a magnetic shielding chamber (three spherical shielding layers with inner diameter of $5 \mathrm{~m}$ ) (Hirao et al. 1985), kindly made available by JAXA at the Institute of Space and Astronautical Science (ISAS).

Each test consisted of three basic phases: first, to check the magnetic DC conditions of MASCOT; second, to check the performance of the MasMag instrument; and third, to measure the magnetic signature of each MASCOT unit under a nominal operational profile. In all the signature tests, MasMag's performance was confirmed as qualitatively comparable to the calibration results in terms of magnetic noise and sensitivity, indicating stable performance and validity of the ground calibration.

The final test revealed an absolute magnitude of the DC bias field of MASCOT at the MasMag sensor position of the order of $2500 \mathrm{nT}$. A detailed investigation showed that this DC bias field is not stable in time. Besides an expected thermal drift, some units cause a change in the DC bias field due to their construction and operation. These include the mobility mechanism, whose stepper motor and magnets on the eccentric arm produce a variable quasi-DC field depending on the arm position. Another instrument is MicrOmega, with its cryocooler possessing a motor with moving magnetic parts. The total DC field changes are in the range of tens of $\mathrm{nT}$ when comparing the DC values observed at the beginning with those at the end of the final magnetic cleanliness test. The variability in the separate components can be even larger, ranging up to hundreds of nT. These changes will influence the MasMag measurement accuracy and need to be closely analyzed by comparison with housekeeping information as well as the on-ground calibration and test data. The required accuracy ( $1 \mathrm{nT})$ given by the scientific objectives is, however, not violated by the DC offset change of the MicrOmega operation, as the MicrOmega operation takes place when MASCOT is stationary on the surface. Relevant measurements done by MasMag for the main objectives are done during descent and hopping. The DC offset change by the mobility mechanism is concentrated on a short timescale (seconds) prior to MASCOT movement and therefore the DC offset can be removed from the data. Moreover, data along the hopping trajectory are of the highest interest for the scientific investigations. In addition, the thermal dependence of the DC offset of the MASCOT unit needs to be accounted for and evaluated.

Important parts of the tests were single-unit signatures in the magnetic field data measured by MasMag. A specific operational sequence was provided for each MASCOT unit in order to have a representative nominal operational scenario of the unit. The tests were successful in characterizing each unit and showing that a clear identification of the artificial signals is possible. As an example, the AC signature of representative CAM operations is shown in Fig. 5. A power-on signature in the magnetic field components is visible in the left panel. The most prominent change, $-43 \mathrm{nT}$, is visible in the $B_{z}$ component. The right panel shows another CAM operation in which a red and a green LED diodes are used. Both profiles are clear and easy to identify in the data.

Almost all of the units have an operational signature that is predictable and can be removed from the MasMag data by post-processing. Moreover, the raw MasMag data provide useful housekeeping information on the overall MASCOT system. A more complex field profile was identified for the MicrOmega instrument, as it involves a cryocooler operation (magnetic parts) and various current consumption profiles that depend on particular measurement settings. However, even such a heavily contaminated magnetic field record can be processed to determine the ambient field on the asteroid surface. Moreover, MicrOmega shall operate in phases that do not correlate with MasMag high-priority phases.

In summary, though MASCOT is a highly integrated system, methods are available to remove the bias field as well as the AC disturbances to guarantee that MasMag can provide scientific measurements within a given overall accuracy of $1 \mathrm{nT}$ in order to fulfill its given objectives. 


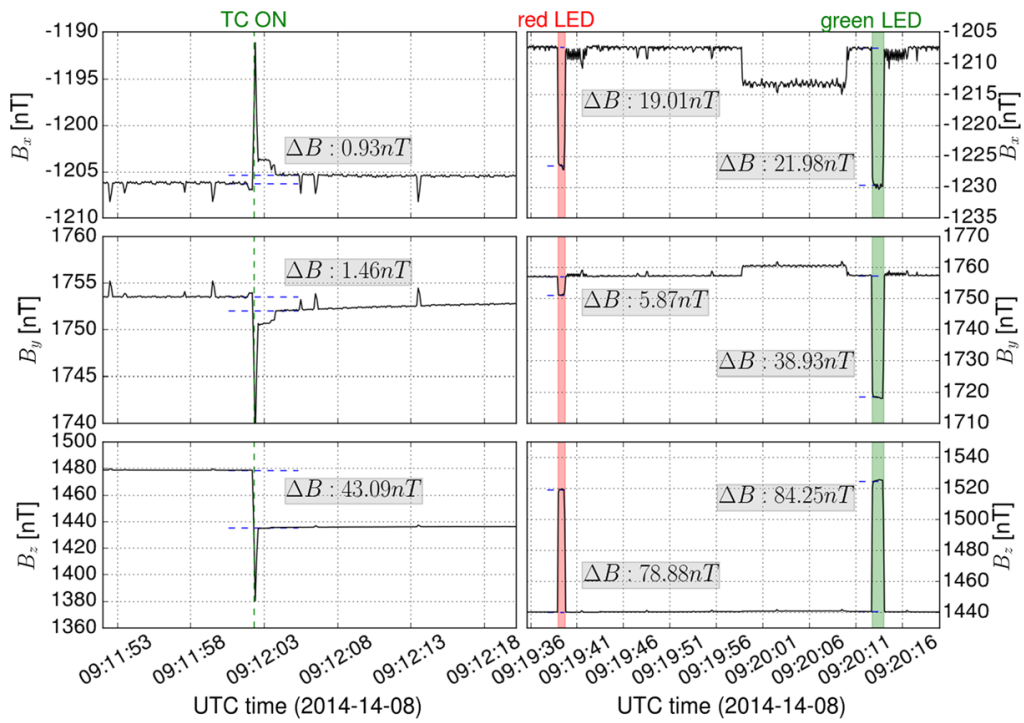

Fig. 5 Example of CAM operations signatures in the magnetic field as observed during the ground testing in the JAXA magnetic shielding facility. The left panels show power ON profile with an overshoot and the strongest signal in the $B_{z}$ component of $-43 \mathrm{nT}$. The right panels show profiles of CAM red and green LED illumination. Both signals are clearly distinguishable

\subsection{Commissioning}

The Hayabusa2 spacecraft was launched on December 3, 2014. The first commissioning and check-outs started shortly after the launch. The MASCOT lander was switched on for the first time in space on December 16, 2014, when a short check-out took place. A health check procedure incorporated various system as well as payload procedures to check the status of the MASCOT lander. All expected data were successfully acquired by the Hayabusa 2 spacecraft from MASCOT and transmitted to the Earth without any packet loss. The next MASCOT activities were a second in-flight health check in June and calibration in September 2015. The housekeeping (HK) values were nominal and indicated no problems, considering the MasMag operation and performance.

As expected, the location within the Hayabusa2 spacecraft body proved to be magnetically not clean. The most prominent feature is a DC offset, large enough to cause a saturation of the $B_{x}$ component of the MasMag instrument in the default measurement mode. However, switching into a different MasMag mode (compensation) with static bias compensation solved the issue, as validated during calibration campaign. The AC disturbances consisted of numerous smaller field jumps (10 to $50 \mathrm{nT}$ ) at 1- to 5-second intervals. Larger disturbances $(\sim 200 \mathrm{nT})$ are present aperiodically. Also, the non-deterministic AC disturbances (background noise) increased significantly by Hayabusa2 activities. Table 4 summarizes the influences on the magnetic field measurements for MASCOT only and within the Hayabusa2 environment with MasMag stand-alone performance. Although the maximum AC values in case of MASCOT are higher than for Hayabusa2 observed so far, the MASCOT interactions are far more sparse, and the maximum value appears only during the MicrOmega operation that is not critical for MasMag science, as discussed above. In-flight calibration campaign was intended to validate ground calibrations and check instrument noise and internal 
Table 4 A comparison on magnetic field disturbances for the stand-alone MasMag instrument, MasMag integrated within MASCOT and for MASCOT in-flight within Hayabusa2 spacecraft. It compares the DC offset, maximal AC disturbances, and the magnetic noise computed at $1 \mathrm{~Hz}$ from $(0.5,1.5) \mathrm{Hz}$ bandwidth. Values given are absolute (sign omitted)

\begin{tabular}{lllll}
\hline Influence type & MasMag & with MASCOT & with Hayabusa2 \\
\hline $\mathrm{DC}[\mathrm{nT}]$ & $\mathrm{B}_{\mathrm{x}}$ & 18 & 1200 & $\sim 12200$ \\
& $\mathrm{~B}_{\mathrm{y}}$ & 15 & 1800 & 8700 \\
& $\mathrm{~B}_{\mathrm{z}}$ & 4 & 1100 & 7200 \\
Deterministic AC $[\mathrm{nT}]$ & $\mathrm{N} / \mathrm{A}$ & $<800$ & $<200$ \\
Non-deterministic AC $[\mathrm{pT} / \sqrt{\mathrm{Hz}} @ 1 \mathrm{~Hz}]$ & $15^{\mathrm{a}}$ & $\sim 40$ & $\sim 200$ \\
\hline
\end{tabular}

${ }^{\mathrm{a}}$ The internal MasMag instrument noise.
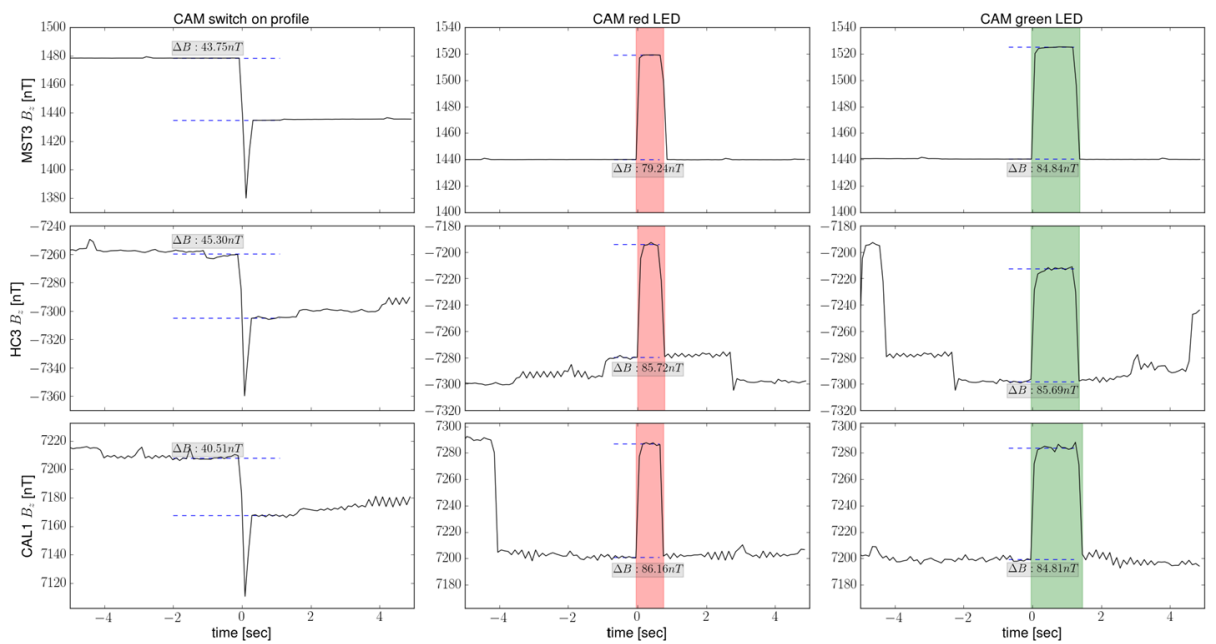

Fig. 6 Comparison of the MasMag measured magnetic field $B_{z}$ component during CAM activities. Left panels: CAM switch-on profile, middle panels: CAM red LED operation, right panels: CAM green LED operation. The data were acquired during different campaigns. First dataset (top panels) is from the ground Magnetic Signature Test 3 (MST3) in JAXA's shielding chamber with MASCOT only. Second row of panels shows data from the first in-flight Health Check (HC3) within Hayabusa2. Last dataset (lower panels) is from the most recent Calibration (CAL1) activity that took place on 17 September 2015

settings, like phase and $\mathrm{k}$-values used for the field computation. The results are satisfactory, only for the internal noise, within the Hayabusa2 environment, could not be properly checked. However, the data are sufficient to demonstrate that the performance of the instrument ensures the fulfillment of the scientific objectives in terms of measurement accuracy. Because the lander will separate from Hayabusa2 at the asteroid, the MasMag scientific operations and goals are not endangered by the magnetic disturbances from the Hayabusa2 spacecraft.

During the MasMag operation, other MASCOT units also went through their status checks and these were used to compare their magnetic signature with the on-ground measurements. Figure 6 shows a comparison of the $B_{z}$ magnetic field component acquired by the MasMag instrument during CAM operation on three ocasions: (1) During the ground Magnetic Signature Test 3 (MST3) performed in a shielding chamber in JAXA (top pan- 
els). (2) Data from first in-flight Health Check (middle panels). (3) Data from MASCOT Calibration campaign (lower panels). Three activities performed by CAM were chosen for the comparison, namely CAM switch-on (left panels), red LED operation (middle panels), and green LED operation (right panels). As the ground test results showed (see Fig. 5), the $B_{z}$ component experiences most prominent changes, therefore, we show this component only. The comparison shows deterministic AC disturbances that can be identified in the data. The field jump values are almost the same (within $+/-5 \mathrm{nT}$ level), which provides evidence that the MASCOT magnetic activity profile has not been changed after launch. The results therefore indicate that characterization from the ground tests is valid and can be used for the identification of MASCOT-produced artificial signals in the post-processing of the data.

\section{Operations Plan}

During the cruise phase, that is, between launch and arrival to the asteroid, there will be several switch-on phases of MASCOT to check out the status of each instrument. During these periods, MasMag will undergo a health check and various in-flight calibration procedures. MasMag will also continue to monitor operations of other instruments as it provides valuable HK information on other units' activities.

Scientific operations will start once MASCOT is prepared for separation from the Hayabusa2 spacecraft. The separation will take place $\sim 100 \mathrm{~m}$ above the asteroid surface, lasting for $\sim 30$ minutes. After the free fall and the first touchdown, MASCOT will most probably bounce on the surface before finally coming to rest. The whole landing phase, from prior to the separation until the final rest position, is a high-priority phase for the magnetometer measurements. The descent will provide an opportunity to register any increase in the magnetic field possibly caused by the asteroidal global field. Bouncing will provide more measurements from several points on the surface, revealing the local magnetic state of the asteroid's material.

Similar conditions apply for the relocation of MASCOT. After a full characterization of the first landing site by all the MASCOT instruments, MASCOT will try to hop to another position on the asteroid in order to be able to repeat the measurements, also on a different spot. The observations during the relocation will provide a valuable field profile with respect to the height above the surface.

It has been shown on Philae ROMAP data by Auster et al. (2015) that having a profile from a descent and an ascent trajectory during hopping as well as multiple touchdowns is highly beneficial for the data analysis and the confidence level of the results. In addition, the magnetometer data provided a precise timing for estimation of the touchdowns and Philae trajectory.

Having two magnetometers, one on the surface and one in orbit, proved highly beneficial for increasing the accuracy of the ROMAP measurements and also for the attitude reconstruction (Heinisch et al. 2015). Even if we cannot benefit from having two magnetometers on Hayabusa 2 to provide such a space truth for the MASCOT magnetometer measurements, much information can be yielded even with a single magnetometer. The data analysis can provide information about the rotation of the lander, such as rotation frequencies or abrupt changes in orientation that would indicate the landing/bouncing. Therefore, the magnetometer is not only a valid scientific instrument, but also a valuable engineering tool. 


\section{Summary}

The MASCOT lander will provide in situ characterization of the Hayabusa2 target asteroid-162173 Ryugu. After the Hayabusa2 arrival at the asteroid in 2018 and an initial global mapping and monitoring phase, MASCOT will be released. During the landing and the surface phase, the MASCOT magnetometer will be observing ambient magnetic fields. Focusing on the descent and relocation phases, the magnetic field measurement shall provide valuable information about the asteroid's own magnetic field and the magnetization of the surface material. Although the magnetic cleanliness conditions are not ideal due to the fact that MasMag is placed within the MASCOT lander body, the overall MasMag performance and the detailed MASCOT characterization, that enables a removal of the magnetic field disturbances coming from MASCOT itself within a required accuracy of $1 \mathrm{nT}$, ensures the scientific return of the mission. The enhanced ground test campaign provided enough information about MASCOT magnetic properties. This gathered information will be used to clean and calibrate final scientific data in order to derive magnetic properties of the asteroid 162173 Ryugu.

Acknowledgements The lead investigator group at the Technische Universität Braunschweig is financially supported by the Deutsches Zentrum für Luft- und Raumfahrt and the Bundesministerium für Wirtschaft und Energie under contract 50OW1203. The MasMag team would like to acknowledge extensive support and help during integration and testing from the DLR MASCOT system team lead by Tra-Mi Ho. BPW would like to thank Thomas F. Peterson and the NASA Emerging Worlds program for support.

Open Access This article is distributed under the terms of the Creative Commons Attribution 4.0 International License (http://creativecommons.org/licenses/by/4.0/), which permits unrestricted use, distribution, and reproduction in any medium, provided you give appropriate credit to the original author(s) and the source, provide a link to the Creative Commons license, and indicate if changes were made.

\section{References}

M.H. Acuña, B.J. Anderson, C.T. Russell, P. Wasilewski, G. Kletetschka, L. Zanetti, N. Omidi, NEAR magnetic field observations at 433 eros: first measurements from the surface of an asteroid. Icarus 155(1), 220-228 (2002). ISSN:0019-1035. doi:10.1006/icar.2001.6772

V. Angelopoulos, The THEMIS mission. Space Sci. Rev. 141(1-4), 5-34 (2008). ISSN:0038-6308. doi:10. 1007/s11214-008-9336-1

H.-U. Auster, How to measure Earth's magnetic field. Phys. Today 61(2), 76 (2008). ISSN:0031922808023. doi:10.1063/1.2883919

H.-U. Auster, I. Apathy, G. Berghofer, A. Remizov, R. Roll, K.-H. Fornaçon, K.H. Glassmeier, G. Haerendel, I. Hejja, E. Kührt, W. Magnes, D. Moehlmann, U. Motschmann, I. Richter, H. Rosenbauer, C.T. Russell, J. Rustenbach, K. Sauer, K. Schwingenschuh, I. Szemerey, R. Waesch, ROMAP: Rosetta magnetometer and plasma monitor. Space Sci. Rev. 128(1-4), 221-240 (2007). doi:10.1007/s11214-006-9033-X

H.-U. Auster, K.H. Glassmeier, W. Magnes, O. Aydogar, W. Baumjohann, D. Constantinescu, D. Fischer, K.-H. Fornaçon, E. Georgescu, P. Harvey, O. Hillenmaier, R. Kroth, M. Ludlam, Y. Narita, R. Nakamura, K. Okrafka, F. Plaschke, I. Richter, H. Schwarzl, B. Stoll, A. Valavanoglou, M. Wiedemann, The THEMIS fluxgate magnetometer. Space Sci. Rev. 141(1-4), 235-264 (2008). doi:10.1007/s11214-0089365-9

H.-U. Auster, I. Richter, K.H. Glassmeier, G. Berghofer, C.M. Carr, U. Motschmann, Magnetic field investigations during ROSETTA’s 2867 Šteins flyby. Planet. Space Sci. 58(9), 1124-1128 (2010). doi:10.1016/ j.pss.2010.01.006

H.-U. Auster, I. Apathy, G. Berghofer, K.-H. Fornaçon, A. Remizov, C. Carr, C. Güttler, G. Haerendel, P. Heinisch, D. Hercik, M. Hilchenbach, E. Kührt, W. Magnes, U. Motschmann, I. Richter, C.T. Russell, A. Przyklenk, K. Schwingenschuh, H. Sierks, K.H. Glassmeier, The nonmagnetic nucleus of comet 67P/Churyumov-Gerasimenko. Science (2015). doi:10.1126/science.aaa5102

J. Biele, S. Ulamec, Capabilities of Philae, the Rosetta lander. Space Sci. Rev. 138(1-4), 275-289 (2008). doi:10.1007/s11214-007-9278-Z 
X. Blanco-Cano, Hybrid simulations of solar wind interaction with magnetized asteroids: comparison with Galileo observations near Gaspra and Ida. J. Geophys. Res. 108(A5), 1216 (2003). doi:10.1029/ 2002JA009618

T.H. Burbine, T.J. McCoy, A. Meibom, B. Gladman, K. Keil, Meteoritic parent bodies: their number and identification, in Asteroids III, vol. 1, ed. by W.F. Bottke, A. Cellino, P. Paolicchi, R.P. Binzel (University of Arizona Press, Tucson, 2002), pp. 653-667

H. Campins, J. de León, A. Morbidelli, J. Licandro, J. Gayon-Markt, M. Delbo, P. Michel, The origin of asteroid 162173 (1999 JU3). Astron. J. 146(2), 26 (2013). doi:10.1088/0004-6256/146/2/26

L. Carporzen, B.P. Weiss, L.T. Elkins-Tanton, D.L. Shuster, D. Ebel, J. Gattacceca, Magnetic evidence for a partially differentiated carbonaceous chondrite parent body. Proc. Natl. Acad. Sci. USA 108(16), 63866389 (2011). doi:10.1073/pnas. 1017165108

A. Coradini, F. Capaccioni, S. Erard, G. Arnold, M.C. De Sanctis, G. Filacchione, F. Tosi, M.a. Barucci, M.T. Capria, E. Ammannito, D. Grassi, G. Piccioni, S. Giuppi, G. Bellucci, J. Benkhoff, J.P. Bibring, A. Blanco, M. Blecka, D. Bockelee-Morvan, F. Carraro, R. Carlson, U. Carsenty, P. Cerroni, L. Colangeli, M. Combes, M. Combi, J. Crovisier, P. Drossart, E.T. Encrenaz, C. Federico, U. Fink, S. Fonti, L. Giacomini, W.H. Ip, R. Jaumann, E. Kuehrt, Y. Langevin, G. Magni, T. McCord, V. Mennella, S. Mottola, G. Neukum, V. Orofino, P. Palumbo, U. Schade, B. Schmitt, F. Taylor, D. Tiphene, G. Tozzi, The surface composition and temperature of asteroid 21 Lutetia as observed by Rosetta/VIRTIS. Science 334(6055), 492-494 (2011). doi:10.1126/science.1204062

C. Cournede, J. Gattacceca, M. Gounelle, P. Rochette, B.P. Weiss, B. Zanda, An early solar system magnetic field recorded in CM chondrites. Earth Planet. Sci. Lett. 410, 62-74 (2015). doi:10.1016/j.eps1.2014.11. 019

L.T. Elkins-Tanton, B.P. Weiss, M.T. Zuber, Chondrites as samples of differentiated planetesimals. Earth Planet. Sci. Lett. 305(1-2), 1-10 (2011). ISSN:0012-821X. doi:10.1016/j.eps1.2011.03.010

C.P. Escoubet, R. Schmidt, M.L. Goldstein, Cluster—science and mission overview. Space Sci. Rev. 79, 1132 (1997). doi:10.1023/A:1004923124586

R.R. Fu, B.P. Weiss, Detrital remanent magnetization in the solar nebula. J. Geophys. Res. 117(E2), 02003 (2012). doi:10.1029/2011JE003925

R.R. Fu, E.A. Lima, B.P. Weiss, No nebular magnetization in the Allende CV carbonaceous chondrite. Earth Planet. Sci. Lett. 404, 54-66 (2014). doi:10.1016/j.epsl.2014.07.014

J. Gattacceca, P. Rochette, Toward a robust normalized magnetic paleointensity method applied to meteorites. Earth Planet. Sci. Lett. 227, 377-393 (2004). ISSN:0012-821X. doi:10.1016/j.eps1.2004.09.013

K.H. Glassmeier, I. Richter, A. Diedrich, G. Musmann, U. Auster, U. Motschmann, A. Balogh, C. Carr, E. Cupido, A. Coates, M. Rother, K. Schwingenschuh, K. Szegö, B. Tsurutani, RPC-MAG the fluxgate magnetometer in the ROSETTA plasma consortium. Space Sci. Rev. 128(1-4), 649-670 (2007). doi:10. 1007/s11214-006-9114-X

K.H. Glassmeier, H.-U. Auster, D. Heyner, K. Okrafka, C. Carr, G. Berghofer, B.J. Anderson, A. Balogh, W. Baumjohann, P. Cargill, U. Christensen, M. Delva, M. Dougherty, K.-H. Fornaçon, T.S. Horbury, E.A. Lucek, W. Magnes, M. Mandea, A. Matsuoka, M. Matsushima, U. Motschmann, R. Nakamura, Y. Narita, H. O’Brien, I. Richter, K. Schwingenschuh, H. Shibuya, J.A. Slavin, C. Sotin, B. Stoll, H. Tsunakawa, S. Vennerstrom, J. Vogt, T. Zhang, The fluxgate magnetometer of the BepiColombo Mercury Planetary Orbiter. Planet. Space Sci. 58(1-2), 287-299 (2010). doi:10.1016/j.pss.2008.06.018

O. Grasset, M.K. Dougherty, A. Coustenis, E.J. Bunce, C. Erd, D. Titov, M. Blanc, A. Coates, P. Drossart, L.N. Fletcher, H. Hussmann, R. Jaumann, N. Krupp, J.P. Lebreton, O. Prieto-Ballesteros, P. Tortora, F. Tosi, T. Van Hoolst, JUpiter ICy moons explorer (JUICE): an ESA mission to orbit Ganymede and to characterise the Jupiter system. Planet. Space Sci. 78, 1-21 (2013). ISSN:0032-0633. doi:10.1016/j.pss. 2012.12.002

P. Heinisch, H.-U. Auster, I. Richter, D. Hercik, E. Jurado, R. Garmier, C. Güttler, K.H. Glassmeier, Attitude reconstruction of ROSETTA's lander PHILAE using two-point magnetic field observations by ROMAP and RPC-MAG. Acta Astronaut. (2015). ISSN:0094-5765. doi:10.1016/j.actaastro.2015.12.002

K. Hirao, K. Tsuruda, I. Aoyama, T. Saito, Large spherical magnetic shield room. J. Geomagn. Geoelectr. 37(6), 581-588 (1985). doi:10.5636/jgg.37.581

M.G. Kivelson, L.F. Bargatze, K.K. Khurana, D.J. Southwood, R.J. Walker, P.J. Coleman, Magnetic field signatures near Galileo's closest approach to Gaspra. Science 261, 331-334 (1993)

T.J. McCoy, M. Gaffey, W.V. Boynton, T.H. Burbine, C.R. Chapman, A. Cheng, P.E. Clark, L.G. Evans, P. Gorenstein, The Composition of 433 Eros: A Mineralogical-Chemical Synthesis, 1661-1672 (2001)

N.F. Ness, K.W. Behannon, R.P. Lepping, N.F. Ness, K.H. Schatten, Use of two magnetometers for magnetic field measurements on a spacecraft. J. Geophys. Res. Space Phys. 76, 3564-3573 (1971). doi:10.1029/ JA076i016p03564

H. Nübold, K.H. Glassmeier, Accretional remanence of magnetized dust in the solar nebula. Icarus 144(1), 149-159 (2000). doi:10.1006/icar.1999.6273 
J. Reill, H.-J. Sedlmayr, P. Neugebauer, M. Maier, E. Krämer, R. Lichtenheldt, MASCOT—asteroid lander with innovative mobility mechanism, in ASTRA 2015 (2015)

I. Richter, D.E. Brinza, M. Cassel, K.H. Glassmeier, F. Kuhnke, G. Musmann, C. Othmer, K. Schwingenschuh, B.T. Tsurutani, First direct magnetic field measurements of an asteroidal magnetic field: DS1 at Braille. Geophys. Res. Lett. 28(10), 1913-1916 (2001). doi:10.1029/2000GL012679

I. Richter, H.-U. Auster, K.H. Glassmeier, C. Koenders, C.M. Carr, U. Motschmann, J. Müller, S. McKennaLawlor, Magnetic field measurements during the ROSETTA flyby at asteroid (21) Lutetia. Planet. Space Sci. 66(1), 155-164 (2012). doi:10.1016/j.pss.2011.08.009

M. Terho, L.J. Pesonen, I.T. Kukkonen, Magnetic properties of asteroids from meteorite data-implications for magnetic anomaly detections. Earth Moon Planets 72, 225-231 (1996). doi:10.1007/BF00117522

N.J. Turner, S. Fromang, C. Gammie, H.H. Klahr, G. Lesur, M. Wardle, X. Bai, Transport and accretion in planet-forming disks, in Protostars Planets VI, vol. 1, ed. by H. Beuther, R.S. Klessen, C.P. Dullemond, T.K. Henning (2014), pp. 411-432. doi:10.2458/azu_uapress_9780816531240-ch018

J. Veverka, B. Farquhar, M. Robinson, P. Thomas, S. Murchie, A. Harch, P.G. Antreasian, S.R. Chesley, J.K. Miller, W.M. Owen, B.G. Williams, D. Yeomans, D. Dunham, G. Heyler, M. Holdridge, R.L. Nelson, K.E. Whittenburg, J.C. Ray, B. Carcich, A. Cheng, C. Chapman, J.F. Bell, M. Bell, B. Bussey, B. Clark, D. Domingue, M.J. Gaffey, E. Hawkins, N. Izenberg, J. Joseph, R. Kirk, P. Lucey, M. Malin, L. McFadden, W.J. Merline, C. Peterson, L. Prockter, J. Warren, D. Wellnitz, The landing of the NEARShoemaker spacecraft on asteroid 433 Eros. Nature 413(6854), 390-393 (2001). doi:10.1038/35096507

B.P. Weiss, J. Gattacceca, S. Stanley, P. Rochette, U.R. Christensen, Paleomagnetic records of meteorites and early planetesimal differentiation. Space Sci. Rev. 152(1-4), 341-390 (2010). doi:10.1007/s11214-0099580-z

T.L. Zhang, G. Berghofer, W. Magnes, M. Delva, W. Baumjohann, H. Biernat, H. Lichtenegger, MAG: the fluxgate magnetometer of venus express. ESA SP 1295, 1-10 (2007) 